\title{
Game-based cache allocation strategy for ICN slicing
}

\author{
Liping Ge, and Jinhe Zhou*
}

School of Information and Communication Engineering, Beijing Information Science and Technology University, Beijing 100101, China

\begin{abstract}
To reduce the delay of content acquisition, this paper proposes a game-based cache allocation strategy in the Information-Centric Network (ICN) slice. The cache resource allocation of different mobile virtual network operators (MVNOs) is modeled as a non-cooperative game model. The Newton iterative method is used to solve this problem, and the cache space allocated by the base station for each MVNO is obtained. Finally, the Nash equilibrium solution is obtained. Simulation results show that the proposed algorithm can reduce the delay.
\end{abstract}

\section{Introduction}

The traditional IP addressing method is inefficient for users[1]. In the 5G scenario, slicing can meet the requirements of low delay, high reliability and enough bandwidth. But the related problems in the process of information retrieval have not been solved. ICN technology realizes name-address separation, which can reduce latency, improve resource utilization and cache hit ratio [2]. ICN technology can effectively reduce data transmission delay by caching it near user groups with social relations[3]. However, there are still many problems in the deployment of ICN. 5G architecture has key technologies such as Network Function Virtualization (NFV), network slicing and so on. 5G network implements and maintains an end-to-end network slice instance according to the service requirements and network status[4]. Akihiro Nakao et al. [5] introduces the preliminary research work to realize 5G mobile end-to-end network slicing. R. Ravindran et al. [6] introduces a 5G-ICN architecture, compares it with innovative services supporting ICN functions and the current mobile architecture based on 3GPP, and gives an in-depth overview of resource allocation, interdependence and coordination between 5G-ICN functional slices. H. Jin et al. [7] introduces an Information-Centric virtual content network(IVCN) slicing framework. From the perspective of information centric networking and service provision, a method for dividing virtual network functions(VNF) is proposed. The question of how to deploy ICN in the network has been studied above, but the research on deploying cache in a slice is still lacking. Deployment of cache resources in network slicing can improve content delivery and end-user experience quality [8]. Q. Jia et al. [9] studied the cache resource allocation

* Corresponding author: zhoujinhe@bistu.edu.cn 
Scheme of network slicing is studied by combining network caching with network slicing using game theory, which can significantly improve the utilization rate of cache resources. P. L. Vo et al. [10] introduces coordination between the allocation of Ran resources for each chip and the sharing of the same resource slices in a wireless access network (RAN). To sum up, ICN technology can be implemented by slicing and supports cache function, and the game algorithms can solve the effective allocation of cache resources.

\section{Game model of ICN}

In this paper, a cache allocation algorithm based on a non-cooperative game in ICN slice is proposed. MVNO cache allocation in slice is modeled as a non-cooperative game model. It is proved that the game has Nash equilibrium. The Newton iteration method is used to solve the optimal solution to maximize the interests of participants. Fig. 1 illustrates the imaging model of the game.

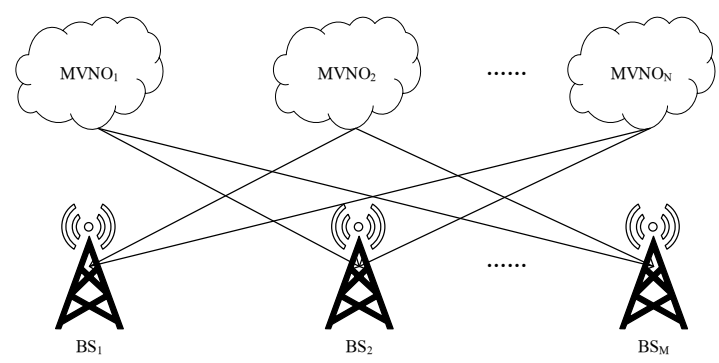

Fig. 1. Game model of ICN.

\subsection{Game construction}

In this paper, the cache allocation of MVNO is modeled as a non-cooperative game. In this model, there are $M$ base stations (BS) $\{1,2, \ldots \ldots, M\}$, the cache capacity of each base station is $\mathrm{C}$, and the user set served by the is $\left\{\mathrm{u}_{1}, \mathrm{u}_{2}, \ldots \ldots, \mathrm{u}_{L}\right\}$. According to the user's delay demand, the base station allocates the cache space to MVNO. It is assumed that each user's allocated bandwidth conforms to orthogonal frequency division multiplexing technology. The bandwidth allocated by the base station for MVNO is $W_{m, n}$, and L is the number of users served by the $M V N O_{n}$.

It is all the bandwidth that the base station can allocate for MVNO

$$
W_{n}=\sum_{m=1}^{M} \sum_{l=1}^{L} W_{m, n, l}
$$

When providing services for $M V N O_{n}$, the signal to interference noise ratio between base station $m$ and user $l$ is

$$
Y_{m, n, l}=\frac{p_{m, n} g_{m, n, l}}{\sigma^{2}+\sum_{n_{i} \neq n} p_{m, n_{i}} g_{m, n_{i}, l}}
$$


$\sigma^{2}$ is the channel noise, and $\sum_{n_{i} \neq n} p_{m, n_{i}} g_{m, n_{i}, l}$ is the interference from the same base station serving other MVNOs. From this, we can get the sum of instantaneous transmission rate of $M V N O_{n}$ serving users is

$$
R_{n}=W_{n} \log _{2}\left(1+\sum_{m=1}^{M} \sum_{l=1}^{L} Y_{m, n, l}\right)
$$

The arrival probability of content request follows the Zipf distribution. $x_{m, n}$ is the cache space provided by the base station for $M V N O_{n}$. For one MVNO, the cache space of all base stations is

$$
\begin{gathered}
x_{n}=\sum_{m=1}^{M} \beta_{m} x_{m, n} \\
\beta_{m}=\int_{0}^{x_{m, n}} \frac{c}{f^{-\alpha}} d f=\left(\frac{x_{m, n}}{\kappa_{n}}\right)^{1-\alpha}
\end{gathered}
$$

Then the transmission delay of $M V N O_{n}$ is

$$
\begin{aligned}
& D_{n}=D_{\text {core }}-\kappa_{n}^{2-\alpha} \sum_{m=1}^{M} \frac{x_{m, n}^{2-\alpha}}{W_{m, n} \log _{2}\left(1+Y_{n}\right)} \\
& \text { s.t } x_{m, n} \leq C
\end{aligned}
$$

\subsection{Game optimization}

MVNO will play games because of the limited cache space of the base station, and its purpose is to maximize its own interests. Nash equilibrium is the solution of the non-cooperative game and the stable state of the game among the players. Assuming that each player can make a rational decision, when the game reaches Nash equilibrium, the utility of each player will be maximized, and each party participating in the game can not obtain more benefits by unilateral change of strategy. However, the optimal solution does not necessarily exist. Some non-cooperative game models do not have such solutions, while some models may have multiple such solutions.

Theorem 1 The strategy set of $M V N O_{n}$ is $\left\{x_{m, n}\right\}$, there is a non-cooperative game between MVNOs, if $D\left(x_{m, n}^{*}\right) \geq D\left(x_{m, n}\right)$, there is a Nash equilibrium $x_{m, n}^{*}$.

Proof The Nash equilibrium is satisfied if the second partial derivative satisfies the concave function property.

$$
\frac{\partial^{2} D_{n}}{\partial^{2} x_{m, n}}=\sum_{m=1}^{M} \frac{-\mathrm{K} x_{m, n}^{-\alpha}}{W_{m, n} \log _{2}\left(1+Y_{n}\right)} \leq 0
$$

It can be concluded that the second-order partial derivative satisfies the concave function characteristic. That proves the existence of Nash equilibrium point. The proof is over. Each 
MVNO always has a Nash equilibrium point, and can always find a set of optimal cache strategies to maximize its utility.

\section{Algorithm solution}

To simplify and solve the optimization model, Newton iterative algorithm is used to solve the problem, which can realize the reasonable allocation of resources in a cache. The basic principle is to assume that all base stations at time $t$ have made cache decisions. When users send requests to MVNO, they can get the best response according to their utility function. Based on the analysis in the previous section, we will use the Newton iteration method to solve this problem. The iterative formula for cache resource allocation is

$$
x_{m, n}(t+1)=x_{m, n}(t)+\delta \frac{\partial D_{n}}{\partial x_{m, n}(t)}
$$

\section{Simulation results and analysis}

In this paper, matlab R2018b is used to simulate the algorithm. In order to optimize the cache resource allocation, only two base stations are considered. $\alpha=0.3$, $C=500 M B, p_{m, n}=10 \mathrm{~W}, L=20, W_{m, n, l}=10 \mathrm{KHZ}$.

As can be seen in Fig. 2, with the increase of iterations, the cache allocated for each MVNO tends to a fixed value, that is, Nash equilibrium solution.

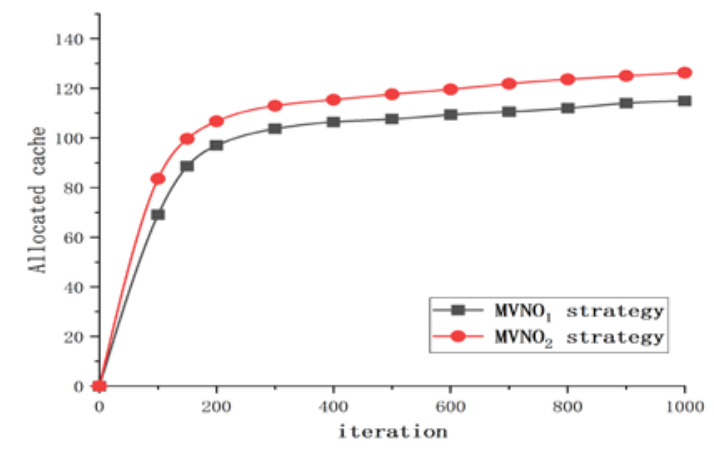

Fig. 2. Allocated cache of MVNO.

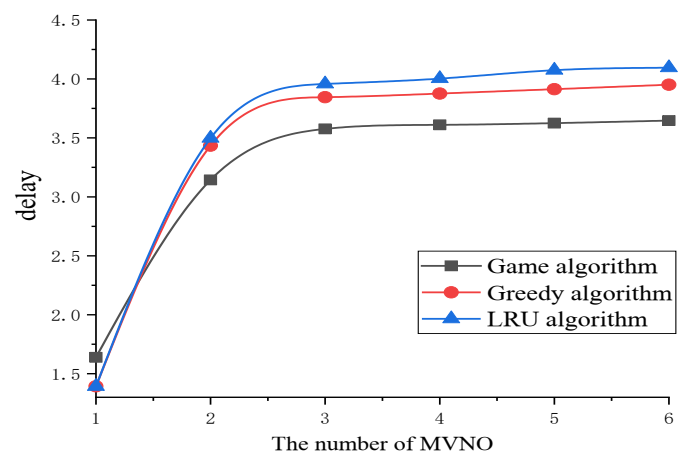

Fig. 3. Delay variation of different MVNO numbers. 
The curve in Fig. 3 shows that the delay increases with the increase of MVNO number for a given number of base stations. Because multiple MVNOs will lead to more requests, the base station can not provide adequate cache for MVNOs, resulting in increased delay.

\section{Conclusion}

To meet the different needs of different services, $5 \mathrm{G}$ network introduces network slicing technology. The cache resource allocation of multiple MVNOs is modeled as a non-cooperative game, and the Newton Raphson algorithm is used to solve the cache allocation. This algorithm can reduce the transmission delay.

This work is supported by the National Natural Science Foundation of China "Research on Intelligent Dynamic Resource Allocation and Optimization Methods for 5G Ultra-Dense Access Networks" (61872044).

\section{References}

1. Z. Zhang, C. Lung, M. St-Hilaire and I. Lambadaris, "Smart Proactive Caching: Empower the Video Delivery for Autonomous Vehicles in ICN-Based Networks," in IEEE Transactions on Vehicular Technology, 69, 7 (2020) 7955-7965.

2. J. Duan, Y. Xing, G. F. Zhao. Survey for caching technologies in information centric networking. Com-puter Engineering andApplications, 54, 2 (2018) 1-10.

3. X. Zhang, Q. Zhu. Information-Centric Virtualization for Software-Defined Statistical QoS Provisioning Over 5G Multimedia Big Data Wireless Networks. IEEE Journal on Selected Areas in Communications, J. 37, 8 (2019) 1721-1738.

4. H. Xiang, S. Yan, M. Peng. A Realization of Fog-RAN Slicing via Deep Reinforcement Learning. IEEE Transactions on Wireless Communications, J. 19, 4 (2020) 2515-2527.

5. Akihiro Nakao,Ping Du,Yoshiaki Kiriha,Fabrizio Granelli,Anteneh Atumo Gebremariam,Tarik Taleb,Miloud Bagaa. End-to-end Network Slicing for 5G Mobile Networks. Journal of Information Processing, J. 25 (2017)

6. R. Ravindran, A. Chakraborti, S. O. Amin, A. Azgin and G. Wang, "5G-ICN: Delivering ICN Services over 5G Using Network Slicing," in IEEE Communications Magazine, 55, 5 (2017) 101-107.

7. H. Jin, H. Lu, Y. Jin and C. Zhao, "IVCN: Information-Centric Network Slicing Optimization Based on NFV in Fog-Enabled RAN," in IEEE Access, 7 (2019) 69667-69686.

8. Q. Zhao, L. Feng, S. Guo, P. Yu and F. Zhou, "Joint Caching Allocation and Delay Control for Network Slices Carrying Multimedia Services in 5G Core Network," 2019 IEEE International Symposium on Broadband Multimedia Systems and Broadcasting (BMSB), Jeju, Korea (South), (2019) 1-6.

9. Q. Jia, R. Xie, T. Huang, J. Liu and Y. Liu, "Efficient caching resource allocation for network slicing in 5G core network," in IET Communications, 11, 18 (2017) 2792-2799.

10. P. L. Vo, M. N. H. Nguyen, T. A. Le and N. H. Tran, "Slicing the Edge: Resource Allocation for RAN Network Slicing," in IEEE Wireless Communications Letters, 7, 6 (2018) 970-973. 